\title{
Nonlinear Modelling of Kinetic Data Obtained from Photocatalytic Mineralisation of 2,4-Dichlorophenol on a Titanium Dioxide Membrane
}

\author{
Ignazio Renato Bellobono, ${ }^{1}$ Roberto Scotti, ${ }^{2}$ Massimiliano D'Arienzo, ${ }^{2}$ \\ Franca Morazzoni, ${ }^{2}$ Riccardo Bianchi, ${ }^{3}$ Rodica Stanescu, ${ }^{4}$ Cristina Costache, ${ }^{4}$ \\ Liliana Bobirica, ${ }^{4}$ Gabriela Cobzaru, ${ }^{4}$ Paola Maria Tozzi, ${ }^{5}$ Mauro Rossi, ${ }^{6}$ \\ Mauro Luigi Bonardi, ${ }^{6}$ Flavia Groppi, ${ }^{6}$ and Luigi Gini ${ }^{6}$ \\ ${ }^{1}$ Environmental Research Centre, University of Milan, via C. Golgi 19, 20133 Milan, Italy \\ ${ }^{2}$ Department of Materials Science, University of Milano Bicocca, via R. Cozzi 53, 20126 Milan, Italy \\ ${ }^{3}$ ISTM, Institute of Molecular Sciences and Technologies, CNR, 20133 Milan, Italy \\ ${ }^{4}$ Department of Inorganic Technology and Environmental Protection, Polytechnic University of Bucharest, 011061 Bucharest, Romania \\ ${ }^{5}$ RぬD Group, BIT srl, 20121 Milan, Italy \\ ${ }^{6}$ LASA, Department of Physics, University of Milan, National Institute of Nuclear Physics (INFN), 20133 Milan, Italy
}

Correspondence should be addressed to Ignazio Renato Bellobono, i.r.bellobono@bitechnologies.it

Received 16 October 2008; Revised 11 December 2008; Accepted 26 January 2009

Recommended by Peter Robertson

Photomineralisation of 2,4-dichlorophenol (DCP) in aqueous solutions (10.0-100.0 mg/L of C) was systematically studied at $318 \pm 3 \mathrm{~K}$, in an annular laboratory-scale reactor, by photocatalytic membranes immobilizing titanium dioxide, as a function of substrate concentration, and absorbed power per unit length of membrane. Kinetics of both substrate disappearance, to yield intermediates, and total organic carbon (TOC) disappearance, to yield carbon dioxide, were followed (first series of experiments). At a fixed value of irradiance $\left(1.50 \mathrm{~W} \cdot \mathrm{cm}^{-1}\right)$, other series of mineralization experiments were repeated (second series of experiments) by carrying out only analyses of chemical oxygen demand (COD), in order to compare modelling results of the two sets of experiments. In both sets of experiments, stoichiometric hydrogen peroxide was used as oxygen donor. For the first series of experiments, a kinetic model was employed, already validated in previous work, from which, by a set of differential equations, four final optimised parameters, $k_{1}$ and $K_{1}, k_{2}$ and $K_{2}$, were calculated. By these parameters, the whole kinetic profile could be fitted adequately. The influence of irradiance on $k_{1}$ and $k_{2}$ could be rationalised very well by this four-parameter kinetic model. Modelling of quantum yields, as a function of irradiance, could also be carried out satisfactorily. As has been found previously for other kinds of substrates, modelling of quantum yields for DCP mineralization is consistent with kinetics of hydroxyl radicals reacting between themselves, leading to hydrogen peroxide, other than with substrate or intermediates leading finally to carbon dioxide, paralleled by a second competition kinetics involving superoxide radical anion. For the second series of experiments, on the contrary, the Langmuir-Hinshelwood model was employed. Uncertainties of COD analyses, coupled with discrepancies of this model and with its inability to reproduce kinetics up to complete mineralization, are underlined.

Copyright (C) 2009 Ignazio Renato Bellobono et al. This is an open access article distributed under the Creative Commons Attribution License, which permits unrestricted use, distribution, and reproduction in any medium, provided the original work is properly cited.

\section{Introduction}

Among the advanced oxidation processes, which were developed during the last twenty five-thirty years, photocatalysis [1-3] in the presence of semiconductors, such as titanium dioxide, with UV radiation, is certainly one of the most studied. If the UV radiation is from the sun, the treatment costs are reduced drastically, by transforming photocatalysis in one of the most appealing and environmentally friendly processes for complete mineralisation of organic pollutants. Furthermore, photocatalytic processes may be used as a pre-treatment of toxic chemicals, in order to convert them 
into fully biodegradable compounds to be finally processed by the most economical technology presently available, that of active sludge [4]. Combination of photocatalysis with active sludge techniques, particularly if solar energy is directly or indirectly employed, is undoubtedly one of the most promising technologies to degrade and mineralise, efficiently and inexpensively, wastes containing toxic compounds.

From the point of view of mechanisms of surface photochemical reactions that take place in heterogeneous systems, a recent article [5] has amply criticised the a priori assumed validity of the Langmuir-Hinshelwood kinetic model to interpret the experimental observations as well as the assertion that the kinetics of a heterogeneous photoreaction are either first-order or half-order dependent on photon flow. Such a criticism has already been emphasized in literature also from the point of view of engineering applications and dates back to earlier pilot plant studies [6]. These studies have shown, for example, the influence of flow rate on apparent thermodynamic constant of adsorption/desorption, relative to the LangmuirHinshelwood model as well as the inadequacy of this model to fit kinetics of mineralisation, other than in the segment of the initial rate [7-10]. This has been evidenced particularly when using immobilised photocatalysts. Consequently, this pseudo-thermodynamic parameter of the LangmuirHinshelwood model, being also linked to reactor geometry and fluid dynamics, should have a, partly at least, kinetic character.

Comparison of efficiency of different kinds of supported or immobilized photocalysts and objective characterisation of their photocatalytic power are thus the major problems to define kinetic reactivity. In most of literature works, this kind of assessment is often made simply by matching qualitatively concentration profiles of degraded species. In addition, and unfortunately, in many cases described in literature, no regard is made to total organic carbon (TOC) mineralisation, but merely to transformation of substrate into some intermediate compound, for example, bleaching of a dye, as the first step of the complicated reaction sequence leading finally to carbon dioxide. Moreover, it is a common practice, even when quantitative evaluations are made, to consider the photocatalytic process as a first-order reaction. On the contrary, it has been definitely ascertained that apparent reaction order varies with concentration of substrate $[6,8]$, with an evident transition from a limiting zero-order, at high concentrations, to a limiting first-order at low concentrations. Modelling of photocatalytic reactions, oppositely, in a wide range of concentrations, and over all the kinetic concentration profile of TOC, leading to full mineralisation, has been approached systematically in previous papers of this series $[11,12]$, by using a fourparameter kinetic model. This has been done also from the stand-point of quantum yields and energy efficiencies, a very important aspect, which is often neglected in the literature pertinent to photocatalysis. For this latter aspect, variation of quantum yields with irradiance and concentration of substrate was taken specifically into consideration [11, $12]$.
Photocatalytic degradation of 2,4-dichlorophenol (DCP) has been widely studied in literature [13-24], mainly because DCP is a key intermediate in the synthesis of the herbicide 2,2-dichlorophenoxyacetic acid, the thermal or bio-oxidative decomposition which may give rise to polychloro dioxins. DCP is consequently classified as a toxic, nonbiodegradable compound [25], which may cause some pathologies and changes to endocrine systems of humans [26, 27]. Among the photocatalytic degradation studies of DCP, kinetic models, both of Langmuirian type $[15-17,20]$, or modified, for example, in the form of a four-parameter kinetic model $[23,24]$, have been used and/or developed, in order to express dependency of rates on concentration of substrate, able to reproduce, as closely as possible, experimental data in more or less broad concentration ranges.

In the present paper, nonlinear modelling, previously employed for methane, as a model molecule of aliphatic hydrocarbons, and for phenol, as a model molecule of aromatics, [11, 12], has been extended to kinetic runs relative to photocatalytic mineralisation of DCP in aqueous solution, as a model molecule of polychloro aromatics. A laboratory-scale membrane reactor was employed, with the main purpose of assessing and comparing results of the four-parameter kinetic modelling with those of the standard Langmuir model, in the perspective of establishing a reliable standardization method of the photocatalytic performance of materials. A particular goal of the work was also to highlight the problems encountered when trying to compare data obtained from TOC measurements to those obtained from chemical oxygen demand (COD) measurements. These latter, being easier and less expensive, are often used to determine the efficacy of wastewater treatments. Therefore, we deemed useful also to evaluate treatment of data obtained by these two analytical methods.

\section{Experimental}

2.1. Materials. 2,4-Dichlorophenol (DCP) was obtained from Aldrich (purity greater than 99.5\%). It was used as received with no further purification. Ultrapure water (maximum contents of $\mathrm{Na}^{+}$and heavy metal ions 0.02 and $0.004 \mathrm{mg} \mathrm{Kg}^{-1}$, resp.) was used for preparation of its solutions. Concentrations of DCP, expressed as carbon, were varied in the range $10-100 \mathrm{mg} \cdot \mathrm{L}^{-1}$. No buffer system was added to solutions, either initially or during photodegradation. Initial $\mathrm{pH}$ was 6.2-6.7, and it dropped to no lower than 2.5 when $\mathrm{HCl}$ content, produced from chlorinated phenol, was greater than $95 \%$ with respect to equivalent initial concentration of substrate, at the highest concentration examined.

2.2. Photocatalytic Membranes. The photocatalytic membranes (PHOTOPERM BIT/313), kindly supplied by B.I.T. srl, Milan, Italy, were standard photocatalytic membranes, immobilising $30 \pm 3 \mathrm{wt} . \%$ of titanium dioxide (P25 by Degussa, Germany). These membranes were prepared (patents pending) by grafting and graftpolymerising, onto a nonwoven microporous polypropylene tissue, $250 \pm 9 \mathrm{~g} \cdot \mathrm{m}^{-2}$ of a prepolymeric blend containing 
$30 \pm 3$ wt. $\%$ of titanium dioxide, under rheological control, in the presence of proprietary photoinitiating and photosensitising systems.

2.3. Apparatus and Procedures. The laboratory-scale photoreactor was substantially the same as that already described [28], with some differences in the operating procedures specified later on. Stoichiometric hydrogen peroxide was used as the oxygen donor. As it has been found in all preceding works of the present series [6-12], at low values of absorbed power density, hydrogen peroxide allowed to attain maximum quantum yields, thus suppressing any contribution of charge carriers recombination. Overall volume of solution being treated was $24.0 \pm 0.08 \mathrm{~L}$; the ratio between the overall reacting volume and the length of the lamp, corresponding to the length of the irradiated side of the membrane, was $160 \pm 6 \mathrm{~cm}^{3} / \mathrm{cm}$. A high pressure mercury arc lamp with a nominal power of $1.00 \mathrm{~kW}$ was employed, kindly supplied by Chimia Prodotti e Processi (Muggiò, Milan, Italy); it was further provided with a stepby-step power regulation device, so that power absorbed by the membrane, as determined actinometrically, could be varied between 0.10 and $4.0 \mathrm{~W} \cdot \mathrm{cm}^{-1}$. Radiating power per unit surface of membrane was preferably expressed by radiating power per unit length, in $\mathrm{W} \cdot \mathrm{cm}^{-1}$ units, as has been done in all preceding works of this series, instead of using $\mathrm{W} \cdot \mathrm{cm}^{-2}$ units, because the membrane was concentric to the lamp. Consequently, the transformation of the latter into the former units involves the membrane circumference. Furthermore, also for engineering purposes, the nominal irradiance of lamps is given, for a cylindrical geometry, in $\mathrm{W} \cdot \mathrm{cm}^{-1}$ units. This allows to obtain directly the fraction of power density, which is effectively absorbed by the membrane, on which quantum yields may be based. Several sets of experiments, each one at a certain value of absorbed power per unit length of membrane, were thus carried out, every one by varying the concentrations of DCP within each set, in the range $10-100 \mathrm{mg} \cdot \mathrm{L}^{-1}$, as stated above. The membrane being concentric to the lamp in the photoreactor used, absorbed power per unit length of lamp was also the same, if referred to unit length of membrane. At $1.50 \mathrm{~W} \cdot \mathrm{cm}^{-1}$ of absorbed power per unit length of the membrane, the overall power absorbed by the membrane, within the absorption range of immobilized semiconductor, in the experimental conditions of the present work, was $150 \mathrm{~W}$, corresponding to $1.26 \times 10^{-2}$ Einstein $\cdot \mathrm{min}^{-1}$. As was done in all preceding papers of this series, concerning laboratory-scale experiments carried out in the presence of hydrogen peroxide as oxygen donor, the radiating flow was filtered by a suitable borosilicate glass. Thus, the radiation wavelengths ranged from the band gap of $\mathrm{TiO}_{2}$ downwards to $315 \mathrm{~nm}$. Consequently, direct photolysis of hydrogen peroxide to give hydroxyl radicals was inhibited. Owing to the refrigeration system of the lamp, by means of water circulating in the lamp sheath, made of quartz, mean temperature, during the runs, was $318 \pm 3 \mathrm{~K}$.

In the present as well as in previous papers of this series, membranes were placed and fixed coaxially with the lamp, in such a way that the flow was perpendicular to the membrane. Owing to the microporous structure of the membrane, the pressure drop was very modest; it corresponded to 0.9$1.6 \mathrm{~mm} \mathrm{Hg}$, when the flow rate of the liquid system was $4 \mathrm{~m}^{3} / \mathrm{h}$ in the laboratory-scale photoreactor.

The disappearance of DCP, as such, was followed by a high performance liquid chromatograph (HPLC Finnigan P4000) equipped with a UV detector, by using a reversephase separation column (Restek Pinnacle II, d-C18 $4.6 \mathrm{~mm}$ $\times 250 \mathrm{~mm}, 5 \mu \mathrm{m})$ and acetonitrile:water:acetic acid (68:30:2) as the mobile phase.

Besides the rate of DCP disappearance, as such, the overall rate of organic carbon disappearance was also followed, in this series of experiments, by total organic carbon (TOC) analysis. To this purpose, a Shimadzu TOC-V instrument was used, by which the contribution of inorganic carbon present was always evaluated, in order to obtain TOC by difference from total carbon and inorganic carbon determinations. Determinations were precise within $20 \mathrm{ppb}$ C.

A second series of experiments was also carried out, in which the rate of DCP disappearance, as such, was not measured. In this series, the overall decrease of organic compounds, either substrate or intermediates, was followed by determination of chemical oxygen demand (COD), in order to compare these sets of data with those relative to the direct measurement of TOC disappearance. Furthermore, contrarily to the first series of experiments cited above, in which absorbed power was varied in each set of experiments, in this second series absorbed power was fixed and kept constant at $150 \mathrm{~W}(1.5 \mathrm{~W} / \mathrm{cm})$. In all experiments of this kind, the overall reacting volume was $4.00 \pm 0.06 \mathrm{~L}$.

During all the duration of the runs, no significant decrease of activity of the immobilized photocatalyst was observed, as checked both by random repetition of experiments at various initial concentrations of DCP and by reiteration of whole series of tests at the beginning and at the end of the experimentation period.

2.4. Kinetic Modelling. Modelling kinetic data for most of experiments, that is those for which the rate of DCP disappearance was measured together with the rate of TOC disappearance, the same procedure described in preceding papers $([23,24]$ and particularly [11]) was closely followed, by using the four-parameter model illustrated with all details in these works.

In this model, mineralisation of substrate $\mathrm{S}$ to $\mathrm{CO}_{2}$ is supposed to occur through one single intermediate $\mathbf{I}$, following

$$
\mathbf{S} \longrightarrow \mathbf{I} \longrightarrow \mathrm{CO}_{2}
$$

and if both $\mathbf{S}$ and $\mathbf{I}$ show a competitive apparent adsorption onto the immobilized semiconductor surface, as expressed by apparent adsorption constants $K_{1}$ and $K_{2}$, respectively, regression equations for experimental data are not expressed in a closed form equation:

$$
y_{(\text {calc })}=f(x),
$$




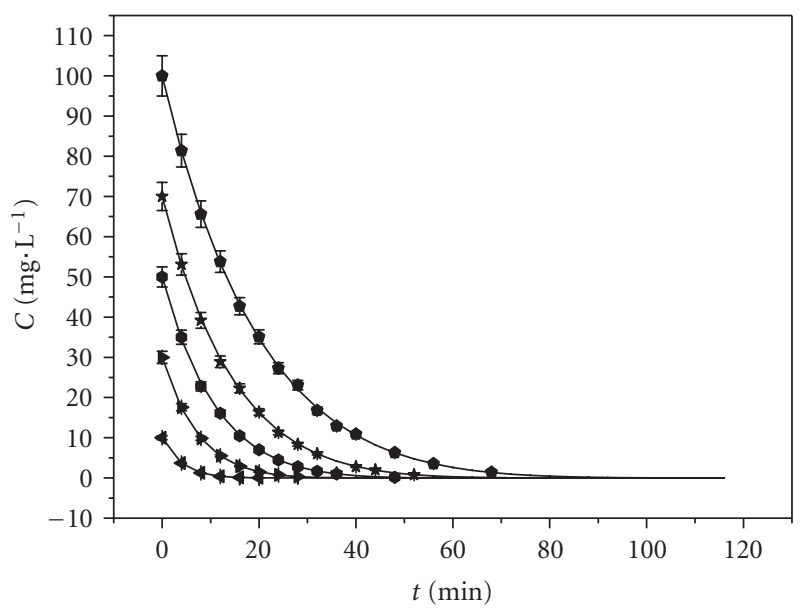

$$
\begin{array}{ll}
k_{1}=5.86(8)(\mathrm{ppm} / \mathrm{min}) & K_{1}=0.077(3)\left(\mathrm{ppm}^{-1}\right) \\
k_{2}=1.06(4)(\mathrm{ppm} / \mathrm{min}) & K_{2}=0.077(4)\left(\mathrm{ppm}^{-1}\right)
\end{array}
$$

FIGURE 1: Kinetics of disappearance of 2.4-dichlorophenol (DCP), from its aqueous solutions, in a laboratory-scale photoreactor (see Experimental), with $1.50 \mathrm{~W} \cdot \mathrm{cm}^{-1}$ of absorbed radiation power per unit membrane length, and standard photocatalytic membranes, immobilising $30 \mathrm{wt} . \%$ of P-25 titanium dioxide. Concentrations, $C$ in $\mathrm{mg} / \mathrm{L}$ (ppm; mass/volume), for DCP, are expressed as carbon, which was not transformed into intermediates, as a function of time $t$, expressed in minutes. In the figure, the four optimised parameters, $k_{1}$ and $K_{1}, k_{2}$ and $K_{2}$, obtained by kinetic modelling, are also reported, together with the probable error, shown between parentheses, as uncertainty in the last digit.

but as the following system of first-order ordinary differential equations in the dependent variables $C_{\mathrm{S}}, C_{\mathrm{I}}$, and $C_{\mathrm{CO}_{2}}$ :

$$
\begin{aligned}
\frac{d C_{\mathrm{S}}}{d t} & =-\frac{k_{1} \cdot K_{1} \cdot C_{\mathrm{S}}}{1+K_{1} \cdot C_{\mathrm{S}}+K_{2} \cdot C_{\mathrm{I}}}, \\
\frac{d C_{\mathrm{I}}}{d t} & =\frac{k_{1} \cdot K_{1} \cdot C_{\mathrm{S}}-k_{2} \cdot K_{2} \cdot C_{\mathrm{I}}}{1+K_{1} \cdot C_{\mathrm{S}}+K_{2} \cdot C_{\mathrm{I}}}, \\
\frac{d C_{\mathrm{CO}_{2}}}{d t} & =\frac{k_{1} \cdot K_{1} \cdot C_{\mathrm{I}}}{1+K_{1} \cdot C_{\mathrm{S}}+K_{2} \cdot C_{\mathrm{I}}},
\end{aligned}
$$

where $C$ denotes concentrations of species to which the pertinent suffix refers, $t$ time; $k_{1}$ and $k_{2}$ the kinetic constants relative to degradation of $\mathbf{S}$ and $\mathbf{I}$, respectively. Intermediate I is supposed to simulate the behaviour of all the often uncountable, numerous intermediates, which are on the route from the substrate $\mathbf{S}$ to $\mathrm{CO}_{2}$. Experimentally speaking, only kinetic profiles of $\mathbf{S}$ and $\mathrm{CO}_{2}$ have to be followed. By solving these equations, together with considerations concerning the overall carbon balance [11], the four parameters, $k_{1}$ and $K_{1}, k_{2}$ and $K_{2}$, may be obtained, by optimisation of all experimental data, as described in previous work $[11,12]$.

Two examples of experimental data, relative to the series of experiments carried out at the value of $150 \mathrm{~W}$ of overall absorbed power, corresponding to $1.26 \times 10^{-2}$ Einstein $/ \mathrm{min}$, are collected in Figures 1 and 2. Figure 1 regards the variation of DCP concentration, expressed as carbon, with time, for all the initial concentrations of DCP examined in the present

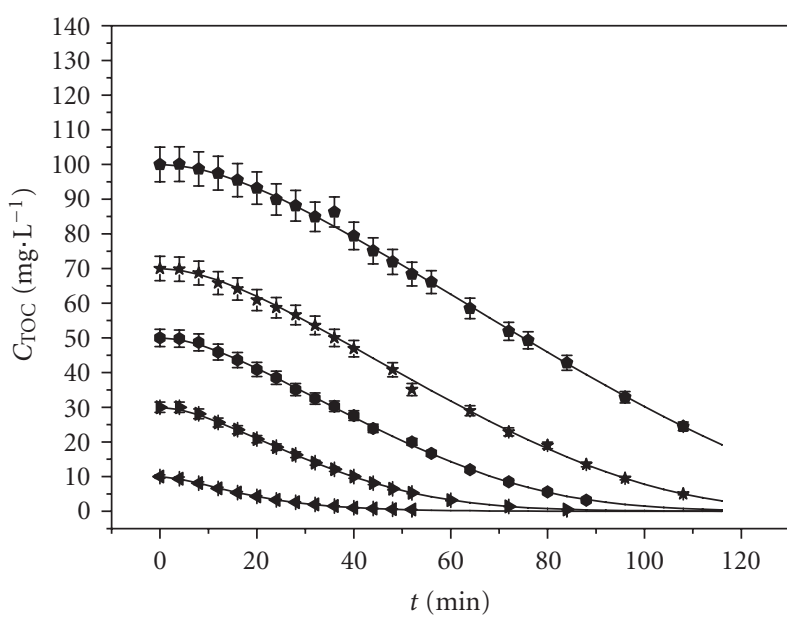

$$
\begin{array}{ll}
k_{1}=5.86(8)(\mathrm{ppm} / \mathrm{min}) & K_{1}=0.077(3)\left(\mathrm{ppm}^{-1}\right) \\
k_{2}=1.06(4)(\mathrm{ppm} / \mathrm{min}) & K_{2}=0.077(4)\left(\mathrm{ppm}^{-1}\right)
\end{array}
$$

Figure 2: Kinetics of disappearance of total organic carbon (TOC) from 2,4-dichlorophenol (DCP) aqueous solutions. TOC is expressed as concentration, $C_{\mathrm{TOC}}$ in $\mathrm{mg} / \mathrm{L}$ (ppm; mass/volume) of carbon, which was not mineralised, as a function of time $t$, expressed in min. Absorbed radiation power per unit membrane length was $1.50 \mathrm{~W} \cdot \mathrm{cm}^{-1}$ during these runs. In the laboratoryscale photoreactor (see Experimental) standard photocatalytic membranes, immobilising $30 \mathrm{wt} \%$ of P-15 titanium dioxide, were employed. In the Figure the four optimised parameters, $k_{1}$ and $K_{1}$, $k_{2}$ and $K_{2}$, obtained by kinetic modelling, are also reported for this series of runs, together with the probable error, shown between parentheses, as uncertainty in the last digit.

paper. Figure 2 regards the variation of TOC with time, for all the corresponding kinetics. All the experimental points of the corresponding couples of single curves reported in Figures 1 and 2 (at equal initial concentrations) were subjected to the optimisation procedure described above. This optimisation gave rise to four parameters, $k_{1}$ and $K_{1}, k_{2}$ and $K_{2}$, for each couple of curves. These parameters differed for each couple only within the uncertainty of the treatment, as a proof that the optimisation method was satisfactory. By extending this treatment to all the couples of Figures 1 and 2, the mean values of the four optimised parameters could be calculated, able to yield the continuous calculated curves. These values are reported inside Figures 1 and 2 themselves. For each value of the optimised parameters, the probable error is equally indicated, between parentheses, in the same figures, shown as uncertainty of the last digit.

On the contrary, for the series of experiments in which only COD was determined, COD data were first transformed into TOC data, by supposing that the ratio between total organic compound, as expressed by COD, and carbon itself was the same as that which was present in DCP. This is already an uncertainty factor, since intermediates do not surely have the same $\mathrm{C} / \mathrm{H}$ ratio as that of the substrate. An example of this kind of kinetics is shown in Figure 3, relatively to the initial concentration of DCP equal to $53 \mathrm{ppm}$ (mass/volume, i.e., $\mathrm{mg} \cdot \mathrm{L}^{-1}$ ). From this sort of graphs, the 


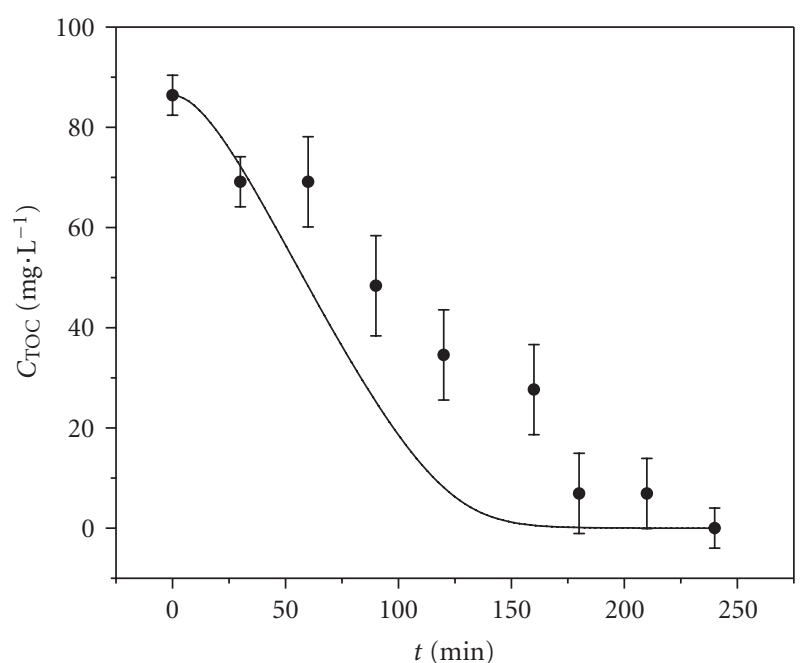

FIgure 3: TOC concentrations, $C_{\text {TOC }}$, in $\mathrm{mg} \cdot \mathrm{L}^{-1}$, obtained by calculation from COD measurements, as a function of time $t$, in minutes, for a kinetic run, starting from a DCP concentration of $84 \mathrm{ppm}$, expressed as carbon, during which measurements of the decrease of organic substances, either substrate or intermediates, versus time $t$, expressed in minutes, was measured by the COD method. Absorbed radiation power per unit membrane length was $1.50 \mathrm{~W} \cdot \mathrm{cm}^{-1}$ and overall reacting volume $4.0 \mathrm{~L}$. In the laboratoryscale photoreactor (see Experimental), standard photocatalytic membranes, immobilising $30 \mathrm{wt} . \%$ of P-15 titanium dioxide, were employed. The curve reported in this figure is that simulated by the four-parameter kinetic model, used in the present work, at the same initial concentration of DCP, and at the same radiating power (see Table 1).

initial rate $r_{0}$ of TOC disappearance was measured and plotted, for the various runs carried out at different initial concentrations $C_{0}$ of DCP, as the reciprocal of $r_{0}$ as a function of the reciprocal of $C_{0}$, following the well-known LangmuirHinshelwood linear form of the adsorption and reaction mechanism:

$$
1 / r_{0}=(1 / k)+\left(1 / k K C_{0}\right),
$$

where $k$ is the rate constant at infinite concentration of substrate, and $K$ the apparent thermodynamic constant for adsorption/desorption equilibrium of substrate between photocalytic membrane and solution.

An example of the graph resulting from classical equation (4) is reported in Figure 4. From this graph, both $k$ and $K$ could be easily obtained by linear regression analysis, for this series of experiments.

\section{Results and Discussion}

Photomineralisation of DCP in aqueous solutions, and in a concentration range corresponding to $10.0-100.0 \mathrm{ppm}$ (mass/volume, i.e., $\mathrm{mg} \cdot \mathrm{L}^{-1}$ ) of carbon, was studied at 318 $\pm 3 \mathrm{~K}$, in a laboratory-scale reactor. Stoichiometric hydrogen peroxide was used as oxygen donor, and photocatalytic membranes immobilising $30 \pm 3 \%$ of titanium dioxide

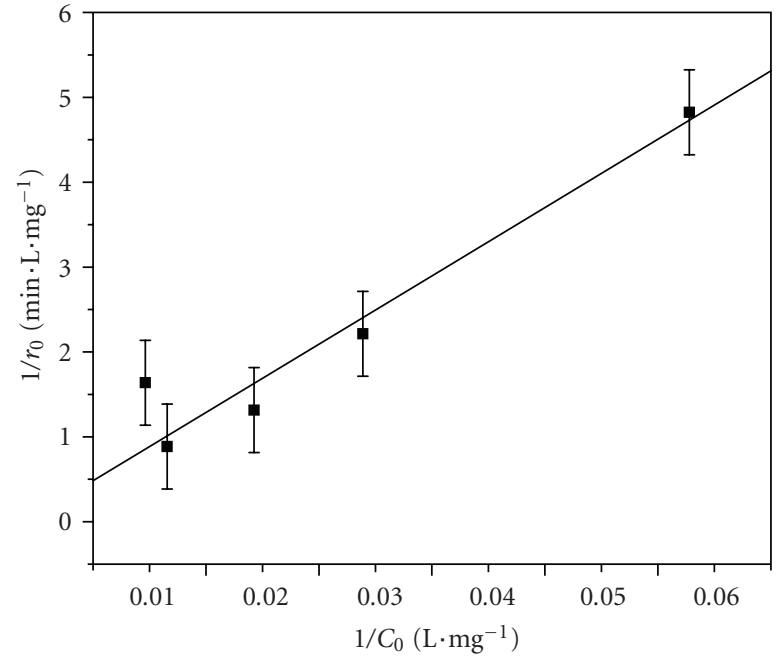

Figure 4: The Langmuir-Hinshelwood plot (4) reporting $\left(1 / r_{0}\right)$ (in $\mathrm{min} \cdot \mathrm{L} \cdot \mathrm{mg}^{-1}$ of mineralised carbon) as a function of $\left(1 / C_{0}\right)$ (in $\mathrm{L} \cdot \mathrm{mg}^{-1}$ of carbon), for all kinetic runs in which analysis has been carried out by COD measurements, these latter being transformed by calculation into total organic carbon. Absorbed radiation power per unit membrane length was $1.50 \mathrm{~W} \cdot \mathrm{cm}^{-1}$ and overall reacting volume $4.0 \mathrm{~L}$, in all the runs of this kind. In the laboratoryscale photoreactor (see Experimental), standard photocatalytic membranes, immobilising $30 \mathrm{wt}$.\% of P-25 titanium dioxide, were employed.

were employed in the photoreactor. Kinetics of both DCP disappearance, to yield intermediates, and total organic carbon (TOC) disappearance, to yield carbon dioxide, were followed. These studies were repeated at various values of overall absorbed power. An example of experimental results of these runs, relative to the set of experiments carried out at an overall value of absorbed power equal to $150 \mathrm{~W}$ $(1.50 \mathrm{~W} / \mathrm{cm})$, is collected in Figures 1 and 2, respectively.

For treating these data, a kinetic model was employed [11], from which, by a set of differential equations (see Experimental), four final optimised parameters, $k_{1}$ and $K_{1}$, $k_{2}$ and $K_{2}$, were calculated. These parameters, by the use of differential equations above, were able to fit the whole kinetic profile very satisfactorily, as shown in the example of Figures 1 and 2. In these Figures comparison is made between experimental data and the continuous curves calculated by the model employed from the values of the four optimised parameters reported in the same figures.

Results relative to all the sets of experiments, carried out as a function of absorbed power per unit length of membrane, are collected in Table 1, in which the four parameters above are reported at the different values of absorbed power per unit length of membrane employed in the variety of experiments.

As it has been remarked and underlined in preceding papers (see particularly $[11,12]), K_{1}$ and $K_{2}$ parameters have very similar values, and for many substrates, as for DCP in the present work, show to coincide, within the limits of experimental uncertainty. On the contrary, they show 
TABle 1: Parameters $\bullet, k_{1}$ and $K_{1}, k_{2}$ and $K_{2}$, able to fit the whole photomineralisation curve, following Scheme (1), at various values of absorbed power per unit length of membrane expressed in $\mathrm{W} \cdot \mathrm{cm}^{-1}$. They were obtained by optimisation of kinetic curves, by using the set of differential equations corresponding to kinetic model of Scheme (1). All the parameters are expressed in ppm of carbon (mass of C/volume, $\mathrm{mg} / \mathrm{L}$ ) and refer to the photodegradation of DCP in aqueous solutions, in the presence of stoichiometric hydrogen peroxide as oxygen donor (treated volume of the aqueous solutions $24.0 \mathrm{~L}$; membrane length $100.0 \pm 0.5 \mathrm{~cm}$; ratio between overall reaction volume and membrane length $160 \pm 6 \mathrm{~cm}^{3} / \mathrm{cm}^{2} ;$ PHOTOPERM BIT/313 membranes immobilising $30 \pm 3 \mathrm{wt} . \% \mathrm{TiO}_{2}$, in the absence of any photopromoter).

\begin{tabular}{lcccc}
\hline $\begin{array}{l}\text { Absorbed power per unit length } \\
\text { of membrane }\left(\mathrm{W} \cdot \mathrm{cm}^{-1}\right)\end{array}$ & $\begin{array}{c}k_{1} \\
(\mathrm{ppm} \mathrm{C} / \mathrm{min})\end{array}$ & $\begin{array}{c}k_{2} \\
(\mathrm{ppm} \mathrm{C} / \mathrm{min})\end{array}$ & $\begin{array}{c}K_{1} \\
(\mathrm{ppm} \mathrm{C})^{-1}\end{array}$ & $\begin{array}{c}K_{2} \\
(\mathrm{ppm} \mathrm{C})^{-1}\end{array}$ \\
\hline 0.15 & $0.821(3)$ & $0.159(3)$ & $0.081(5)$ & $0.076(3)$ \\
0.30 & $1.64(4)$ & $0.321(3)$ & $0.080(4)$ & $0.078(3)$ \\
0.70 & $3.61(5)$ & $0.671(4)$ & $0.076(4)$ & $0.081(3)$ \\
0.90 & $4.32(6)$ & $0.789(4)$ & $0.077(3)$ & $0.075(3)$ \\
1.50 & $5.86(8)$ & $1.06(4)$ & $0.078(5)$ & $0.077(4)$ \\
2.00 & $6.54(9)$ & $1.13(3)$ & $0.077(3)$ & $0.079(3)$ \\
3.00 & $6.49(8)$ & $1.17(6)$ & $0.080(4)$ & $0.078(3)$ \\
4.00 & $6.51(7)$ & $1.15(7)$ & & $0.079(3)$ \\
\hline
\end{tabular}

- Uncertainties are indicated between parentheses and expressed, relatively to the last digits, as probable errors of the means of optimised values from nine sets of runs in the range of concentrations tested.

a marked dependence on flow rate, on reactor geometry, and fluid dynamics $[6,11]$, besides the chemical nature of substrate. Their kinetic behaviour, particularly from the point of view of reactor engineering, stems out undoubtedly. At least, this behaviour is surely shown by $\mathrm{TiO}_{2}$ immobilized in photocatalytic membranes tested. The dependency of $K$ values on chemical character of the corresponding substrates is probably the only remaining aspect of their thermodynamic nature.

Furthermore, Table 1 points out that, while $k_{1}$ and $k_{2}$ appear to be a clear function of irradiance, showing a saturation behaviour at absorbed powers per unit membrane length greater than about $2 \mathrm{~W} \cdot \mathrm{cm}^{-1}, K_{1}$ and $K_{2}$ parameters show an evident independency on irradiance. The kinetic role of substrate and intermediates is adequately and quantitatively highlighted by $k_{1}$ and $k_{2}$ values. For alkanes and alkane-like molecules $[11,29,30] k_{2}$ values are higher than $k_{1}$, and thus the rate is controlled by $k_{1}$, that is, by attack and functionalisation of the starting aliphatic substrate. The contrary occurs for aromatic substrates $[6,8,12,23$, $24]$, the attack of the aromatic ring by hydroxyl radicals being faster than radical reactions involving intermediates, particularly when aliphatic fragments are formed in the way between the starting substrate molecule, and carbon dioxide, as the final product. In the latter cases, consequently, as for DCP in the present work, the rate limiting step is given by transformation of intermediates into carbon dioxide.

An additional and outstandingly important aspect branching from $k_{1}$ and $k_{2}$ values, as well as from $K_{1} \sim K_{2}$ values of Table 1 , is that by using these optimised parameters $[11,12]$, the rates of photomineralisation may be evaluated as a function of initial concentration of substrate, $C_{0}$, and consequently, by operating at a certain value of the photon flow, the corresponding quantum yields $\Phi_{0}$.

As has been found in all previous papers of this series, also for DCP quantum yields $\Phi_{0}$, calculated from rates, followed an apparently Langmuirian function of initial concentration of substrate $C_{0}$, by which $\Phi_{\infty}$ values at "infinite" concentration could be obtained (from the physical point of view, $\Phi_{0}$ values approach $\Phi_{\infty}$ values at a concentration of substrate equal or slightly more than $10^{-3} \mathrm{M}$ )

$$
\frac{1}{\Phi_{0}}=\frac{1}{\Phi_{\infty}}+\frac{1}{\Phi_{\infty} C_{0} K},
$$

where the $K$ value for DCP substantially coincided with $K_{1} \sim K_{2}$ (see Table 1). Finally, by considering all sets of experiments of Table 1, at different values of the absorbed radiant power per unit length of photocatalytic membrane, which was varied in the range $0.10-4.0 \mathrm{~W} \cdot \mathrm{cm}^{-1}$, and by applying (5) to each set of these experiments, the $\Phi_{\infty}$ values as a function of the absorbed radiant power, per unit length of photocatalytic membrane, were obtained, as reported in Figure 5.

This curve of Figure 5 looks very much like those observed in preceding papers of this series (see particularly $[11,12])$. It first shows an initial plateau, up to about $0.4 \mathrm{~W} / \mathrm{cm}$, matching the maximum allowable $\Phi_{\infty}$ value $(0.0417 \mathrm{~mol} /$ Einstein $=1 / 24)$; this value corresponds to a quantitative transformation of one molecule, in the case of DCP, by 24 photons yielding 24 hydroxyl radicals able to mineralise the substrate into chloride ions, carbon dioxide, and water, following exactly the stoichiometric equation. In other words, experimental quantum yields at infinite concentration appear to result in the maximum allowable ones when irradiance is low. Then, quantum yields decrease sharply by increasing irradiance values from about $0.4 \mathrm{~W} / \mathrm{cm}$ to about 2. $-2.5 \mathrm{~W} / \mathrm{cm}$, and much more gently from about $2.5 \mathrm{~W} / \mathrm{cm}$ on. In the case of phenol and methane $[11,12]$, this part of the curve had a very low slope, so that experimental points at values higher than about $2.5 \mathrm{~W} / \mathrm{cm}$ could simulate an apparent plateau.

To explain the behaviour of sigmoid curves, such as that of Figure 5, a competition kinetics has been envisaged 


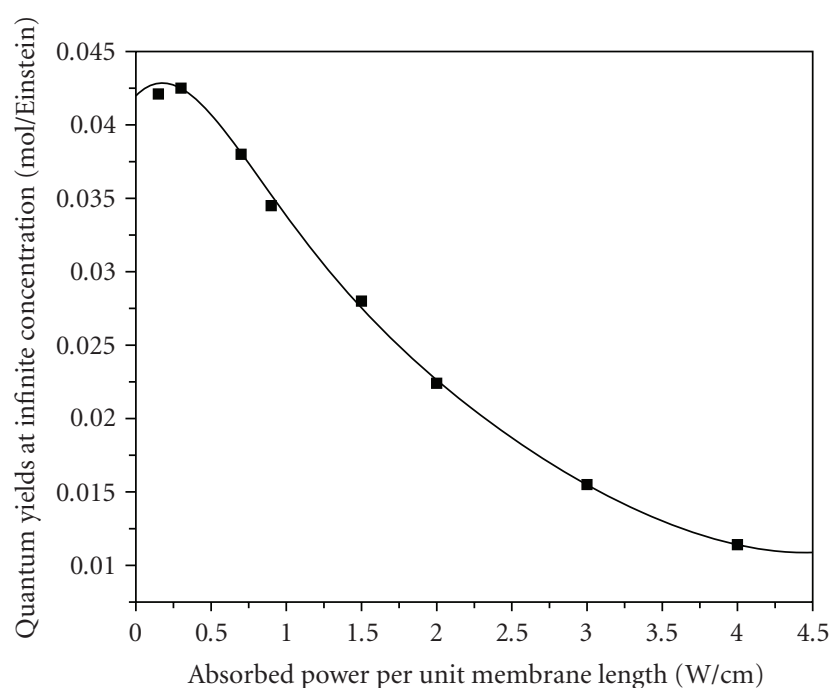

FIgURE 5: Quantum efficiencies $\Phi_{\infty}$ (mol/Einstein) for photocatalytic mineralisation of DCP in aqueous solution, by photocatalytic membranes immobilising titanium dioxide, extrapolated by (5), at "infinite" concentration of substrate, evaluated as mean values (black dots), relative to measurements of kinetic parameters reported in Table 1 , as a function of absorbed power per unit length of irradiated photocatalytic membrane, expressed as $\mathrm{W} \cdot \mathrm{cm}^{-1}$.

$[11,12]$. A reaction of hydroxyl radicals with themselves to give hydrogen peroxide, accompanied by the reaction of these radicals, as well as of superoxide anion radical and/or its conjugate acid, with the substrate and intermediates, leads to mineralisation. These reactions are given by reactions (a) on one side and reactions and (b) and ( $\left.b^{\prime}\right)$ on the other.

Reaction (a) may correspond, as it has been shown [11, 12], to the formation of hydrogen peroxide by recombination of hydroxyl radicals:

$$
\cdot \mathrm{OH}+{ }^{\bullet} \mathrm{OH} \longrightarrow \mathrm{H}_{2} \mathrm{O}_{2} \text {. }
$$

Reaction (b) $[11,12]$ consists in the traditional attack of the substrate by hydroxyl radicals, and may be expressed by

$\bullet \mathrm{OH}+$ substrate $($ or intermediates $) \longrightarrow$ mineraliztion.

Reaction (b') $[11,12]$, involving the $\mathrm{O}_{2}{ }^{\bullet-} / \mathrm{HO}_{2}{ }^{\bullet}$ superoxide radical couple, may be given by

$$
\frac{\mathrm{O}_{2}^{\bullet-}}{\mathrm{HO}_{2}}{ }^{\bullet}+\text { substrate (or intermediates) } \longrightarrow \text { mineralisation. }
$$

This model ignores the possible contribution of charge carriers or surface recombinations. At low irradiance values, when maximum $\Phi_{\infty}$ values are attained, as it has been found in the present and in preceding works, in the presence of hydrogen peroxide as oxygen donor, this contribution is surely negligible. This may not apply equally to high irradiance values. Anyway, modelling above is substantiated, on one side, by quantitative detection of hydrogen peroxide [31] formed onto photocatalytic membranes, when it is not added initially, and on the other side by the compatibility of kinetic data with absolute kinetic constants of reaction (a) $[11,12]$. Therefore, it is not unrealistic to think that onto photocatalytic membranes immobilising titanium dioxide, especially when cocatalysts and hydrogen peroxide are used, scavenging of conduction band electrons is practically quantitative.

To sum up, as regards the $\Phi_{\infty}$ values, also for DCP, the same behaviour that was observed previously, for methane, both in gaseous phase and in aqueous solution, and for phenol in aqueous solution $[11,12]$, appears to be operative. Accordingly, the four-parameter kinetic model, used in the present work as well as in previous papers, performs very well not only from the engineering point of view, which is the first goal to be reached, for practical applications, but also in throwing light onto photocatalytic mechanisms.

Another way is usually employed in literature to reach a reliable characterisation of photocatalytic processes, also in view of its simplicity, notwithstanding the strong criticism, which emerged [5]. This way consists in the application of the two-parameter Langmuir-Hinshelwood model. Consequently, we deemed useful a comparison of results between our model and the classical Langmuir-Hinshelwood equation in its linearised form (4). By operating at the fixed overall adsorbed power of $150 \mathrm{~W}$, corresponding to $1.5 \mathrm{~W} / \mathrm{cm}$ per unit length of membrane, we examined kinetics of photomineralisation, by using the COD method to determine the transformation of organic matter into carbon dioxide (see example of Figure 3). Then, by repeating similar experiments as a function of DCP concentration, the reciprocal of the initial rate of degradation, as given by the slope of the first two or three points in curves such as that of Figure 3, the graph of (4) was plotted in Figure 4, from which the values of $12.5 \pm 0.8 \mathrm{ppm} \cdot \mathrm{min}^{-1}$ for $k$, and of $9.9 \pm 0.9$ for $K$ were obtained.

First of all, one should remark in Figure 3 the great uncertainty of data, as resulting from the means of repeated experiments, which may be attributed to the COD method itself used for following the kinetics. During the runs, the chemical composition of the solution continued to vary, as a consequence of the production of intermediate species. Consequently, the transformation of COD data into total organic carbon values, which is necessary to compare the experiments (based on the assumption that the same ratio between $\mathrm{COD}$ and TOC, that is, the ratio corresponding to the starting substrate, was preserved in the runs notwithstanding the changing of composition), is a rough approximation. This approximation is partly responsible for the lack of confidence in the experimental data. Other reasons for this bearing are surely given by the weariness of the time consuming analytical method, and by its intrinsic difficulties as well as by the great doubt given by the choice of the experimental points to calculate the initial rate. To show the great uncertainty of this latter calculation, in Figure 3 the curve simulated by the four-parameter kinetic model described above is reported, at the same initial concentration of this kinetic run. It may be easily observed that the general trend of the experimental points determined by the COD method 
is very well represented by the four-parameter model. At the same time, the great difference in initial rate clearly appears, either if the slope of the first experimental points is considered to evaluate the initial rate, or if the kinetic criterion should be that of the half transformation time. In both cases an about 30\%-40\% uncertainty results, with respect to the slope of the simulating curve, if the other two criteria of measurements of the photocatalytic activity are used. This, on one side, speaks again in favour of the superior reliability given by the four-parameter model employed in our work; on the other side, this stresses the fact that some experimental points of Figure 3 may lead to deceptive conclusions. Research people should bear in mind these deductions, when reporting, and particularly when comparing, single experiments for assessment of photocatalytic activity. Anyway, by taking into account a congruous number of kinetic runs, and by repeating experimental measurements to estimate the probable error, the correlation factor of the Langmuir-Hinshelwood plot of Figure $4\left(\mathrm{R}^{2}=\right.$ 0.92), almost at the limit of acceptability, allows to assess most conveniently the values of parameters $k$ and $K$ reported above.

Even if parameters $k$ and $K$ of (4) are obtained by a different model with respect to the four-parameter model discussed above, it is interesting to compare their ability and their limits to interpret the two kinds of experiments tested in the present work, those relative to substrate disappearance and TOC measurements, as a function of absorbed power per unit membrane length, and those relative to COD measurements, by (4). As the latter has been carried out at $1.5 \mathrm{~W} / \mathrm{cm}$, only the parameters of the former at the same irradiance value (see Table 1) may be comparable. Furthermore, when comparing $k$ value of the second kind of experiments with $k_{2}$ value of the first kind of experiments, being this step the rate determining one, the $k$ and $k_{2}$ values, expressed in $\mathrm{ppm} \mathrm{C} / \mathrm{min}$, should be converted in $\mathrm{mgC} / \mathrm{min}$, by taking into consideration the different reacting volumes in the two kinds of experiments. A value of $58 \mathrm{mg} \mathrm{C} / \mathrm{min}$ is thus obtained from $k$, and of 25 from $k_{2}$ at an irradiance corresponding to $1.5 \mathrm{~W} / \mathrm{cm}$, both at an infinite concentration value. However, it should be first underlined that consideration on rates, based exclusively on $k$ values, following a Langmuir-Hinshelwood model, should not be regarded as fully trustworthy. This notwithstanding, rough indication based on $k$ values can be considered, at high concentrations of substrate, as complementary to the use of the rate equations (3). This latter is anyway the most consistent method to treat experimental data, as has been done, for example, in kinetic modelling of data reported in Table 1, in which the $k K$ factor shown by (3) may play the most relevant role $[11,12]$. This factor is fully taken into account only when modelling is carried out by (3). Different $K$ and $K_{1}, K_{2}$ values are consequently obtained in Langmuir-Kinshelwood traditional modelling, on one side, and in the four-parameter kinetic modelling of (3), on the other. Only the latter is able to reproduce kinetic data overall the run and not only for initial rates, as the Langmuir-Hinshelwood traditional model does.

\section{Conclusions}

A kinetic model was employed to study photocatalytic mineralisation of DCP in aqueous solution. An annular laboratory-scale membrane reactor, fitted with photocatalytic membranes immobilising titanium dioxide, was used. By a set of differential equations, four final optimised parameters, $k_{1}$ and $K_{1}, k_{2}$ and $K_{2}$, were calculated, able to fit the whole kinetic profile satisfactorily. The influence of irradiance on $k_{1}$ and $k_{2}$ may be thus rationalised.

Quantum yields of mineralisation of DCP in annular photoreactors immobilising the photocatalyst in a membrane structure show a Langmuirian-type dependency on initial concentration of substrate itself. By this relation limiting quantum yields, corresponding to "infinite" concentration may be calculated, together with an apparent thermodynamic constant, coinciding with the lowest between $K_{1}$ and $K_{2}$ values, very often being $K_{1} \sim K_{2}$.

These limiting quantum yields depend on radiant power absorbed per unit membrane length, in the cylindrical geometry of photoreactors. In the low radiant power range, up to about $0.4 \mathrm{~W} / \mathrm{cm}$ they take the shape of a plateau corresponding to the maximum allowable quantum yields, evaluated on the basis of a single hydroxyl radical produced per each absorbed photon, thus showing the excellent performance of the photocatalytic membrane reactors. On the contrary, at high radiant power values, the limiting quantum yields decrease sharply by increasing irradiance values from about $0.4 \mathrm{~W} / \mathrm{cm}$ to about $2 .-2.5 \mathrm{~W} / \mathrm{cm}$, and much more gently from about $2.5 \mathrm{~W} / \mathrm{cm}$ on. In the case of phenol and methane $[11,12]$, this part of the curve had a very low slope, so that experimental points at values higher than about $2.5 \mathrm{~W} / \mathrm{cm}$ could simulate an apparent plateau.

Kinetic parameters of model above have been compared with parameters of the traditional Langmuir-Hinshelwood model. The remarkable uncertainties of the latter model and the fairly greater adequacy of the former were shown in the ability of the four-parameter model to reproduce the whole kinetic profile, up to complete mineralisation of the substrate, and not only in the segment of initial rates, as the Langmuir-Hinshelwood model does.

\section{Acknowledgments}

The present paper is the $83 \mathrm{rd}$ in a series of papers authored by Ignazio Renato Bellobono and his collaborators over the past 22 years which are collectively referred to as the "Photosynthetic Membranes" series. Some of us (R. Stanescu, C. Costache, L. Bobirica, and G. Cobzaru) gratefully acknowledge B.I.T. srl (Milan, Italy), in the network of a permanent agreement with the Polytechnic University of Bucharest, concerning research on B.I.T. patented membranes and processes. G. Cobzaru acknowledges the Polytechnic University of Bucharest, for a grant to carry out research work at the University of Milan and the University of Milano Bicocca. Financial contribution by Fondazione Cariplo to the University of Milano Bicocca is also gratefully acknowledged. 


\section{References}

[1] A. L. Linsebigler, G. Lu, and J. T. Yates Jr., "Photocatalysis on $\mathrm{TiO}_{2}$ surfaces: principles, mechanisms, and selected results," Chemical Reviews, vol. 95, no. 3, pp. 735-758, 1995.

[2] M. R. Hoffmann, S. T. Martin, W. Choi, and D. W. Bahnemann, "Environmental applications of semiconductor photocatalysis," Chemical Reviews, vol. 95, no. 1, pp. 69-96, 1995.

[3] T. L. Thompson and J. T. Yates Jr., "Surface science studies of the photoactivation of $\mathrm{TiO}_{2}$-new photochemical processes," Chemical Reviews, vol. 106, no. 10, pp. 4428-4453, 2006.

[4] I. R. Bellobono, B. Barni, and F. Gianturco, "Preindustrial experience in advanced oxidation and integral photodegradation of organics in potable waters and waste waters by PHOTOPERM $^{\mathrm{TM}}$ membranes immobilizing titanium dioxide and promoting photocatalysts," Journal of Membrane Science, vol. 102, pp. 139-147, 1995.

[5] A. V. Emeline, V. K. Ryabchuk, and N. Serpone, "Dogmas and misconceptions in heterogeneous photocatalysis. Some enlightened reflections," Journal of Physical Chemistry B, vol. 109, no. 39, pp. 18515-18521, 2005.

[6] B. Barni, A. Cavicchioli, E. Riva, et al., "Pilot-plant-scale photodegradation of phenol in aqueous solution by photocatalytic membranes immobilizing titanium dioxide," Chemosphere, vol. 30, no. 10, pp. 1861-1874, 1995.

[7] F. Ascari, I. R. Bellobono, and P. M. Tozzi, "Kinetic modelling of pilot plant photomineralization of aqueous cibacron CR as model molecule of industrial azo dyes on titanium dioxideimmobilizing membranes," Fresenius Environmental Bulletin, vol. 12, no. 10, pp. 1195-1201, 2003.

[8] I. R. Bellobono, F. Ascari, C. Lagrasta, et al., "Kinetic modelling of photomineralization of phenol, as model molecule of aromatic micropollutants, and validation of a photochemical reactor based on photocatalytic membranes immobilizing titanium dioxide and promoting photocatalysts," Fresenius Environmental Bulletin, vol. 12, no. 12, pp. 1536-1544, 2003.

[9] I. R. Bellobono, F. Morazzoni, R. Bianchi, et al., "Laboratoryscale photomineralisation of $n$-alkanes in aqueous solution, by photocatalytic membranes immobilising titanium dioxide," International Journal of Photoenergy, vol. 7, no. 2, pp. 79-85, 2005.

[10] A. Testino, I. R. Bellobono, V. Buscaglia, et al., "Optimizing the photocatalytic properties of hydrothermal $\mathrm{TiO}_{2}$ by the control of phase composition and particle morphology. A systematic approach," Journal of the American Chemical Society, vol. 129, no. 12, pp. 3564-3575, 2007.

[11] I. R. Bellobono, M. Rossi, A. Testino, et al., "Influence of irradiance, flow rate, reactor geometry, and photopromoter concentration in mineralization kinetics of methane in air and in aqueous solutions by photocatalytic membranes immobilizing titanium dioxide," International Journal of Photoenergy, vol. 2008, Article ID 283741, 14 pages, 2008.

[12] I. R. Bellobono, R. Bianchi, G. de Martini, et al., "Nonlinear modelling of data in photomineralization kinetics of organic micropollutants by photocatalytic membranes immobilizing titanium dioxide in membrane reactors," Journal of Chemometrics, vol. 22, no. 7, pp. 425-435, 2008.

[13] Y. Chen, S. Lunsford, and D. D. Dionysiou, "Photocatalytic activity and electrochemical response of titania film with macro/mesoporous texture," Thin Solid Films, vol. 516, no. 21, pp. 7930-7936, 2008.

[14] B. Bayarri, E. Carbonell, J. Giménez, S. Esplugas, and H. Garcia, "Higher intrinsic photocatalytic efficiency of 2,4,6-triphenylpyrylium-based photocatalysts compared to $\mathrm{TiO}_{2}$ P-25 for the degradation of 2,4-dichlorophenol using solar simulated light," Chemosphere, vol. 72, no. 1, pp. 67-74, 2008.

[15] B. Bayarri, M. N. Abellán, J. Giménez, and S. Esplugas, "Study of the wavelength effect in the photolysis and heterogeneous photocatalysis," Catalysis Today, vol. 129, no. 1-2, pp. 231-239, 2007.

[16] B. Bayarri, J. Giménez, D. Curcó, and S. Esplugas, "Photocatalytic degradation of 2,4-dichlorophenol by $\mathrm{TiO}_{2} / \mathrm{UV}$ : kinetics, actinometries and models," Catalysis Today, vol. 101, no. 3-4, pp. 227-236, 2005.

[17] G. Sivalingam, M. H. Priya, and G. Madras, "Kinetics of the photodegradation of substituted phenols by solution combustion synthesized $\mathrm{TiO}_{2}$," Applied Catalysis B, vol. 51, no. 1, pp. 67-76, 2004.

[18] X. Z. Li, B. X. Zhao, and P. Wang, "Degradation of 2,4dichlorophenol in aqueous solution by a hybrid oxidation process," Journal of Hazardous Materials, vol. 147, no. 1-2, pp. 281-287, 2007.

[19] W. A. Adams, M. G. Bakker, T. Macias, and I. A. Jefcoat, "Synthesis and characterization of mesoporous silica films encapsulating titanium dioxide particles: photodegradation of 2,4-dichlorophenol," Journal of Hazardous Materials, vol. 112, no. 3, pp. 253-259, 2004.

[20] C. Chen, P. Lei, H. Ji, et al., "Photocatalysis by titanium dioxide and polyoxometalate $/ \mathrm{TiO}_{2}$ cocatalysts. Intermediates and mechanistic study," Environmental Science and Technology, vol. 38, no. 1, pp. 329-337, 2004.

[21] Y.-C. Chen and P. Smirniotis, "Enhancement of photocatalytic degradation of phenol and chlorophenols by ultrasound," Industrial and Engineering Chemistry Research, vol. 41, no. 24, pp. 5958-5965, 2002.

[22] M. Trillas, J. Peral, and X. Domènech, "Photocatalyzed degradation of phenol, 2,4-dichlorophenol, phenoxyacetic acid and 2,4-dichlorophenoxyacetic acid over supported $\mathrm{TiO}_{2}$ in a flow system," Journal of Chemical Technology and Biotechnology, vol. 67, no. 3, pp. 237-242, 1996.

[23] F. Rota, M. Cavassi, D. Niego, et al., "Mathematical modelling of photomineralization of phenols in aqueous solution, by photocatalytic membranes immobilizing titanium dioxide," Chemosphere, vol. 33, no. 11, pp. 2159-2173, 1996.

[24] L. Tatti, D. Niego, F. Rota, et al., "Mathematical modelling of pilot-plant photomineralization of chlorophenols in aqueous solution, by photocatalytic membranes immobilizing titanium dioxide," Chemosphere, vol. 34, no. 1, pp. 41-49, 1997.

[25] EC Decision 2455/2001/EC of the European Parliament and of the Council, establishing the list of priority substances in the field of water policy and amending Directive 2001/60/EC (L 331 of 13-12-2001), November 2001.

[26] H. Aydın, N. Özdemir, and N. Uzunören, "Investigation of the accumulation of 2,4-dichlorophenoxyacetic acid (2,4-D) in rat kidneys," Forensic Science International, vol. 153, no. 1, pp. 5357, 2005.

[27] J. F. Zhang, H. Liu, Y. Y. Sun, X. R. Wang, J. C. Wu, and Y. Q. Xue, "Responses of the antioxidant defenses of the Goldfish Carassius auratus, exposed to 2,4-dichlorophenol," Environmental Toxicology and Pharmacology, vol. 19, no. 1, pp. 185-190, 2005.

[28] I. R. Bellobono, A. Carrara, B. Barni, and A. Gazzotti, "Laboratory- and pilot-plant-scale photodegradation of chloroaliphatics in aqueous solution by photocatalytic membranes immobilizing titanium dioxide," Journal of Photochemistry and Photobiology A, vol. 84, no. 1, pp. 83-90, 1994. 
[29] I. R. Bellobono, C. Lagrasta, M. L. Carbonara, G. Bonizzoni, and P. M. Tozzi, "Laboratory-scale photomineralization of $n$ alkanols in aqueous solution by photocatalytic membranes immobilizing titanium dioxide," Fresenius Environmental Bulletin, vol. 12, no. 12, pp. 1545-1550, 2003.

[30] I. R. Bellobono, R. Stanescu, C. Costache, et al., "Laboratoryscale photomineralization of $n$-alkanes in gaseous phase by photocatalytic membranes immobilizing titanium dioxide," International Journal of Photoenergy, vol. 2006, Article ID 73167, 8 pages, 2006.

[31] R. Morelli, I. R. Bellobono, C. M. Chiodaroli, and S. Alborghetti, "EPR spin-trapping of hydroxyl radicals onto photocatalytic membranes immobilizing titanium dioxide, and spin adduct competition, as a probe of reactivity with aqueous organic micropollutants," Journal of Photochemistry and Photobiology A, vol. 112, no. 2-3, pp. 271-276, 1998. 


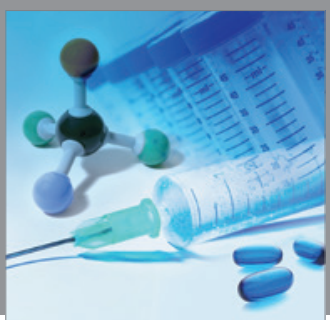

International Journal of

Medicinal Chemistry

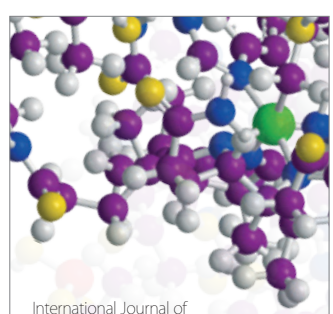

Carbohydrate Chemistry

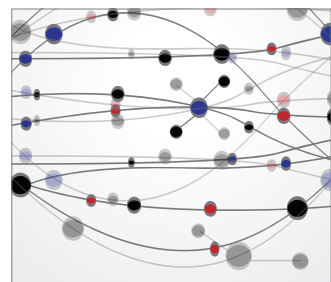

The Scientific World Journal
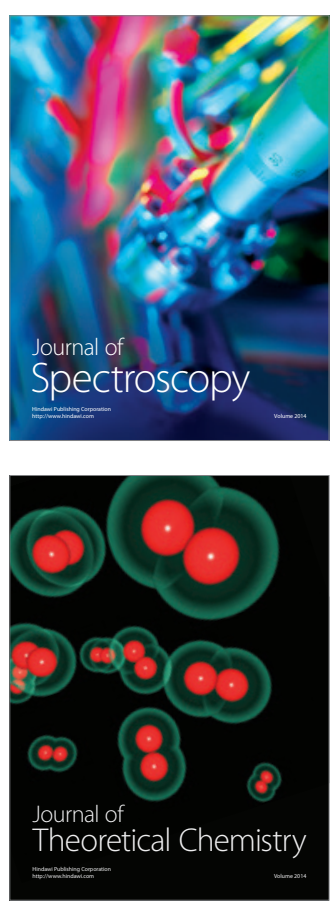
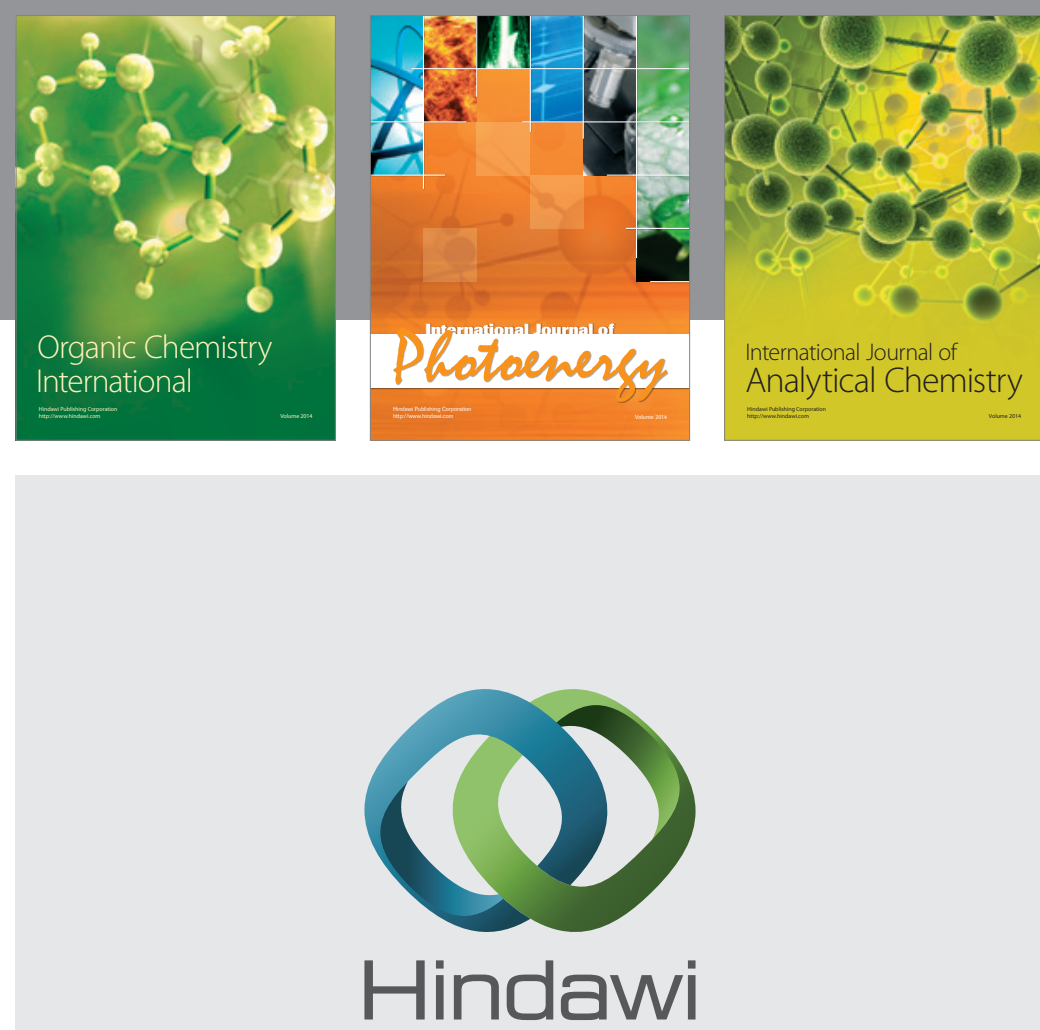

Submit your manuscripts at

http://www.hindawi.com
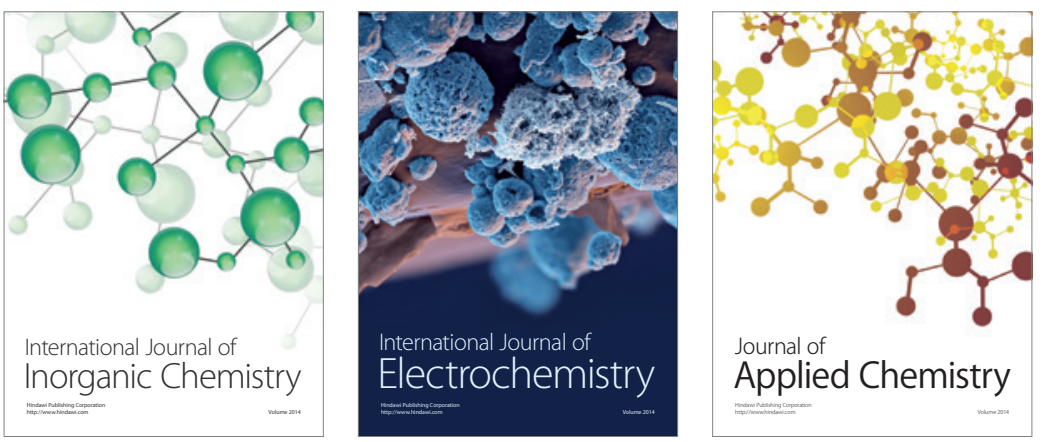

Journal of

Applied Chemistry
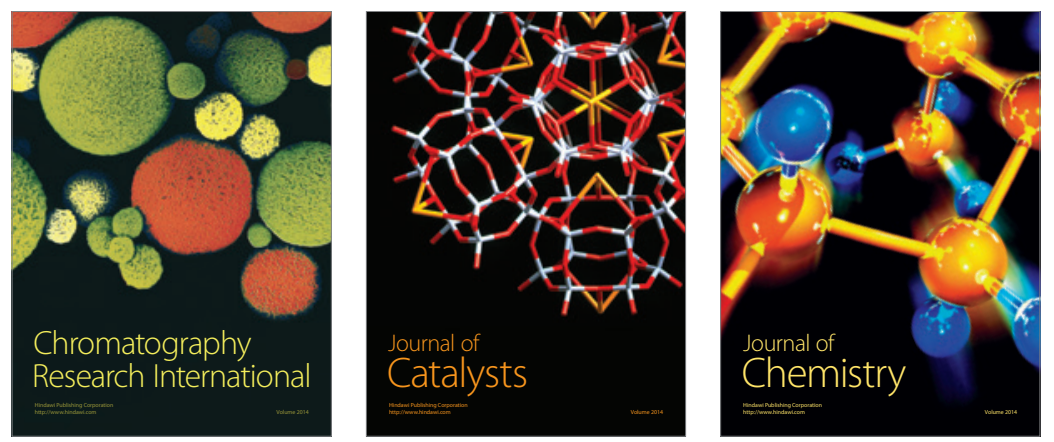
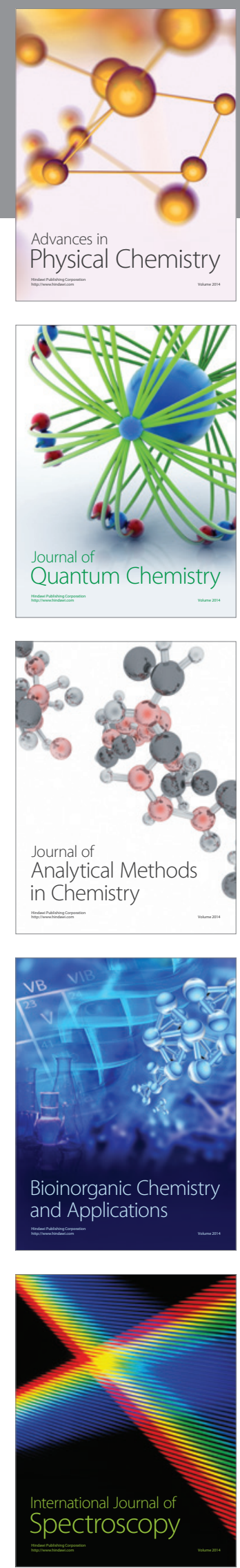